OPEN ACCESS

Edited by:

Benjamin Gesundheit,

Cell El Ltd., Israel

Reviewed by:

Munis Dundar,

Erciyes University, Turkey

Ganesan Venkatasubramanian,

National Institute of Mental Health

and Neurosciences, India

${ }^{*}$ Correspondence:

David Mankuta

mankutad@gmail.com

Specialty section:

This article was submitted to Child and Adolescent Psychiatry,

a section of the journal

Frontiers in Pediatrics

Received: 29 April 2016

Accepted: 13 July 2016

Published: 28 July 2016

Citation:

Gamliel M, Anderson KL, Ebstein RP,

Yirmiya N and Mankuta D (2016)

Paternal HLA-C and Maternal

Killer-Cell Immunoglobulin-Like

Receptor Genotypes in the

Development of Autism.

Front. Pediatr. 4:76.

doi: 10.3389/fped.2016.00076

\section{Paternal HLA-C and Maternal Killer-Cell Immunoglobulin-Like Receptor Genotypes in the Development of Autism}

\author{
Moriya Gamliel', Karen L. Anderson ${ }^{2}$, Richard P. Ebstein ${ }^{3}$, Nurit Yirmiya ${ }^{4}$ and \\ David Mankuta ${ }^{2 *}$
}

${ }^{1}$ Department of Immunology and Cancer Research, Hadassah-Hebrew University Medical Center, Jerusalem, Israel, ${ }^{2}$ Department of Obstetrics and Gynecology, Hadassah-Hebrew University Medical Center, Jerusalem, Israel, ${ }^{3}$ Department of Psychology, National University of Singapore, Singapore, ${ }^{4}$ Department of Psychology, The Hebrew University of Jerusalem, Jerusalem, Israel

Killer-cell immunoglobulin-like receptors (KIRs) are a family of cell surface proteins found on natural killer cells, which are components of the innate immune system. KIRs recognize MHC class I proteins, mainly HLA-C and are further divided into two groups: short-tailed 2/3DS activating receptors and long-tailed 2/3DL inhibitory receptors. Based on the Barker Hypothesis, the origins of illness can be traced back to embryonic development in the uterus, and since KIR:HLA interaction figures prominently in the maternal-fetal interface, we investigated whether specific KIR:HLA combinations may be found in autism spectrum disorders (ASD) children compared with their healthy parents. This study enrolled 49 ASD children from different Israeli families, and their healthy parents. Among the parents, a higher frequency of HLA-C2 allotypes was found in the fathers, while its corresponding ligand 2DS1 was found in higher percentage in the maternal group. However, such skewing in KIR:HLA frequencies did not appear in the ASD children. Additionally, analysis of "overall activation" indicated higher activation in maternal than in paternal cohorts.

Keywords: autism, pregnancy, natural killer, KIR, HLA

\section{INTRODUCTION}

Autism spectrum disorders (ASD) encompass a range of neurodevelopmental syndromes defined by difficulties in social communication and stereotyped behaviors (1). Recent data indicate a prevalence of up to 1 in 66 children (2). Although studies in families and twins (3) point toward a significant genetic component with a high sibling recurrence risk (4), in only a few cases can a clear genetic component be identified. Various factors - hormonal, immunological, and biochemical - have been implicated in autism's etiology, but their roles in the symptomology of the disease remain undefined (5). Immune system dysregulation reported in ASD patients includes differential cell fractions and reactivity, autoimmune phenomena, altered cytokine and antibody profiles, and genetic correlations (6). These findings partially corroborate the theory that chronic neurological inflammation in fetal or newborn brain underlies the development of ASD. Subsequent dysregulation of the immune system may contribute to ASD development in genetically susceptible children.

Abbreviations: ASD, autism spectrum disorders; HLA, histocompatibility leukocyte antigens; KIR, killer-cell immunoglobulinlike receptor; NK, natural killer. 
Altered natural killer (NK) cell activity has been reported in the periphery of ASD patients (7). However, these innate immune cells' function in the pathogenesis of autism is yet unclear. NK cells act specifically against infected or transformed cells, with their actions governed by a balance between activating and inhibitory receptors, each recognizing specific molecules on a given target (8). Killer-cell immunoglobulin-like receptors (KIRs) are a group of cell surface molecules involved in regulating activity of NK (and some T) cells. KIRs are classified as either inhibitory or activating. Their nomenclature is based on the number of extracellular Ig-like domains, and whether the cytoplasmic domain is long (L) or short (S). The KIR gene family is clustered within the LRC leukocyte receptor complex on chromosome 19q13.4, encoding a total of 14 genes and 2 pseudo genes. KIR genes exhibit allelic and haplotypic variability, while each mature NK cell expresses a specific set of KIRs.

Killer-cell immunoglobulin-like receptor counterparts, the HLA class I ligands, are also extremely polymorphic. This generates an additional level of functional diversity. Both KIRs and their HLA ligands are encoded on different chromosomes (19 and 6 , respectively), and therefore segregate independently of each other. This expands the repertoire of possible profiles within any given population (9). Specific KIR-HLA combinations have been suggested as underlying susceptibility as well as resistance to various diseases and conditions (10); the functional implications are less clear. Genetic associations have been reported in infectious diseases (11), autoimmunity (12), inflammatory conditions, reproductive failure, and cancer. Biological models in such studies need to incorporate these receptor:ligand interactions, beyond mere consideration of KIR-HLA genotyping. Indeed, several methods have been proposed to analyze the overall genotyping detected in a specific subject or whole population $(13,14)$.

The KIR family predominantly recognizes HLA class I molecules. Specifically, HLA-C is recognized by both inhibitory receptors (KIRs 2DL1, 2DL2, and 2DL3) and activating receptors (KIRs 2DS1, 2DS2, and 2DS4) (Figure 1). HLA-C group 1 (C1) allotypes have an asparagine residue at position $80\left(\mathrm{C} 1: \mathrm{N}^{80}\right)$, and serve as ligands for KIRs 2DL2, 2DL3, and 2DS2. HLA-C group 2 (C2) allotypes function as ligands for KIRs 2DL1 and 2DS1; C2 allotypes have a lysine at position $80\left(\mathrm{C} 2: \mathrm{K}^{80}\right)$. The interactional affinities of these groups may differ. KIR 2DS4 can bind to both
HLA-C C1 and C2 allotypes, and certain HLA-A alleles. KIRs 3DL1 and (putatively) 3DS1 interact with HLA-A BW4:I80 as well as HLA-B allotypes [with either isoleucine (BW4:I80) or threonine (BW4:T80) at position 80].

The aim of our research was to genotype the KIR receptors known to interact with HLA ligands in autistic children and their non-autistic parents (mothers and fathers, separately), and to compare KIR receptor: HLA ligand frequencies between these groups. Our hypothesis was that a non-random distribution of these genes profiles should be found if interactions between these two classes of molecules are relevant to autism.

According to the Barker Hypothesis (15), the source of pathologies - including mental disorders such as ASD - can be traced back to embryonic existence in the intrauterine environment (16, 17). KIRs are expressed by maternal NK cells (decidua, uterine lining), which are in close contact with HLA-expressing fetal tissues (placenta). Such prenatal interaction between maternal KIR and the fetal HLA in the uterus may lead to either enhanced NK activation or NK inhibition, which in turn may exert influence upon the placenta and/or developing fetus (18-20). Resulting immune activation and effects upon the central nervous system may lead to neurological and psychiatric disorders in predisposed patients. A mouse model for intrauterine maternal immune activation has already been demonstrated as a possible mechanism for autism- and schizophrenia-related behaviors in offspring (21).

\section{MATERIALS AND METHODS}

\section{Participants}

This study comprised of 49 ASD-diagnosed children (38 males, 11 females, a male:female ratio of 3.4:1) from Jewish Israeli families, and their parents. Another six sets of parents of ASD-diagnosed children were enrolled, though no DNA samples were available from their children. DNA samples were collected in Israeli special education schools and treatment centers, by the Israeli Society for Autistic Children (ALUT). ${ }^{1}$

Many of the probands had already been diagnosed with ASD by independent clinicians; two trained clinicians confirmed the

${ }^{1}$ http://www.autismaroundtheglobe.org/countries/Israel.asp
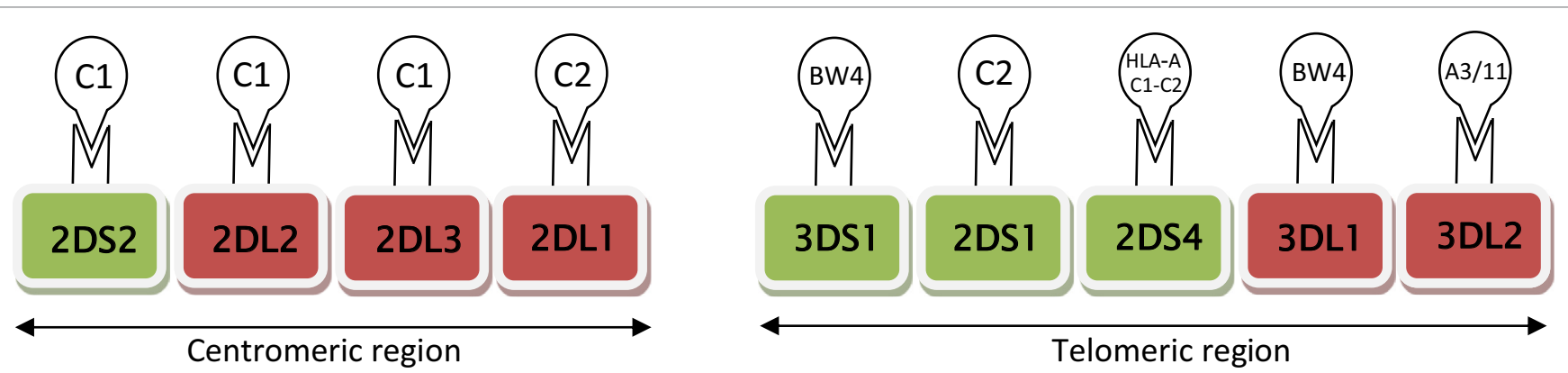

FIGURE 1 | Schematic diagram of activating (green) and inhibitory (red) receptors on natural killer cells, arranged according to centromic/telomeric location. These receptors are known to interact specifically with HLA ligands (HLA Cl or C2, HLA A and B BW4). HLA-C group 1 (Cl) allotypes serve as ligands for KIRs 2DS2, 2DL2, and 2DL3; HLA-C group 2 (C2) functions as ligands for KIRs 2DL1 and 2DS1. 
diagnosis for autism or Pervasive Developmental Disorder-Not Otherwise Specified (PDD-NOS). All probands were diagnosed using the Autism Diagnostic Observation Scale-Generic (ADOS-G) and the Autism Diagnostic Interview-Revised (ADI$\mathrm{R})$. In addition, parents were interviewed using the Vineland Adaptive Behavior Scales-second edition (Vineland ${ }^{\mathrm{TM}}$-II) survey interview edition (VABS).

Probands ranged in age between 25 months to 33 years and 8 months. None of the subjects were known, according to parental reports, to have known genetic factors such as chromosomal aberrations, tuberous sclerosis, or other medical complications that could be related to autism. Parents were screened for psychiatric illness (including ASD); all results were found to be negative.

The Ethics Committee of the Israeli Health Ministry approved this study. All participants signed informed consent forms in advance.

\section{Genotyping}

DNA was extracted from either peripheral blood or buccal cells using a commercial DNA purification kit (Epicentre's Master Pure $^{\mathrm{TM}}$, Madison, WI, USA).

The presence or absence of nine KIR genes known to interact with specific HLA ligands was determined by SSP-PCR (sequence-specific primer polymerase chain reaction), as previously described $(22,23)$, using nine pairs of primers specific for these KIR genes. This mapping enables verification of presence or absence of a KIR, without discriminating specific heterozygous or homozygote status. A 440/750-bp fragment of the CRP/ CAMP, respectively, was included in each PCR as an internal control. The following KIR genes were amplified under identical conditions: 2DL2, 2DL1, 3DL1, 2DS2, 2DS4, and 3DS1. Their
PCR thermo-profile comprised $2 \mathrm{~min}$ at $92^{\circ} \mathrm{C}$; then $25 \mathrm{~s}$ at $91^{\circ} \mathrm{C}$, $45 \mathrm{~s}$ at $65^{\circ} \mathrm{C}$, and $45 \mathrm{~s}$ at $72^{\circ} \mathrm{C}$ for 4 cycles; $25 \mathrm{~s}$ at $91^{\circ} \mathrm{C}, 45 \mathrm{~s}$ at $60^{\circ} \mathrm{C}$, and $45 \mathrm{~s}$ at $72^{\circ} \mathrm{C}$ for 26 cycles; $25 \mathrm{~s}$ at $91^{\circ} \mathrm{C}, 60 \mathrm{~s}$ at $55^{\circ} \mathrm{C}$, and $45 \mathrm{~s}$ at $72^{\circ} \mathrm{C}$ for 5 cycles, and $10 \mathrm{~min}$ at $72^{\circ} \mathrm{C}$ (an extension step, in the case of 3DL1 and 3DS1 was set to $2 \mathrm{~min}$ ). Other KIR amplifications (for 2DL3, 3DL2, and 2DS1) were initiated with denaturation for $2 \mathrm{~min}$ at $92^{\circ} \mathrm{C}$; then $25 \mathrm{~s}$ at $91^{\circ} \mathrm{C}, 45 \mathrm{~s}$ at $57^{\circ} \mathrm{C}$, and $45 \mathrm{~s}$ at $72^{\circ} \mathrm{C}$ for 4 cycles; $25 \mathrm{~s}$ at $91^{\circ} \mathrm{C}, 45 \mathrm{~s}$ at $54^{\circ} \mathrm{C}$, and $45 \mathrm{~s}$ at $72^{\circ} \mathrm{C}$ for 26 cycles; $25 \mathrm{~s}$ at $91^{\circ} \mathrm{C}, 60 \mathrm{~s}$ at $50^{\circ} \mathrm{C}$, and $45 \mathrm{~s}$ at $72^{\circ} \mathrm{C}$ for 5 cycles, and $10 \mathrm{~min}$ at $72^{\circ} \mathrm{C}$. All primer sequences, expected PCR products and allelic recoveries are listed in Table $\mathbf{1 .}$

The distinction of HLA-C KIR ligand groups $\mathrm{C} 1\left(\mathrm{~N}^{80}\right)$ and C2 $\left(\mathrm{K}^{80}\right)$ was also performed by SSP-PCR. The common HLA-C forward primer was either paired with the $\mathrm{C} 1\left(\mathrm{~N}^{80}\right)$ or $\mathrm{C} 2\left(\mathrm{~K}^{80}\right)$ reverse primers, leading to an amplification product of $139 \mathrm{bp}$ (Table 2). This mapping enables discrimination between heterozygous/homozygous status, i.e., $\mathrm{C} 1-\mathrm{C} 1, \mathrm{C} 1-\mathrm{C} 2$, or $\mathrm{C} 2-\mathrm{C} 2$. In each HLA PCR reaction, a 440-bp fragment of the CRP gene was included as an internal PCR control. The thermo-profile comprised an initial denaturation of $3 \mathrm{~min}$ at $95^{\circ} \mathrm{C}$; then $10 \mathrm{~s}$ at $95^{\circ} \mathrm{C}, 30 \mathrm{~s}$ at $65^{\circ} \mathrm{C}$, and $45 \mathrm{~s}$ at $72^{\circ} \mathrm{C}$ for 10 cycles; $10 \mathrm{~s}$ at $95^{\circ} \mathrm{C}, 30 \mathrm{~s}$ at $58^{\circ} \mathrm{C}, 45 \mathrm{~s}$ at $72^{\circ} \mathrm{C}$ for 22 cycles, and $10 \mathrm{~min}$ at $72^{\circ} \mathrm{C}$.

For HLA-C protocol validation, selected samples $(n=30 ; 10$ homozygous $\mathrm{C} 1 / \mathrm{C} 1,10$ homozygous $\mathrm{C} 2 / \mathrm{C} 2$ and 10 heterozygous $\mathrm{C} 1 / \mathrm{C} 2$ ) that had previously been typed for HLA-C at high resolution by LIFECODES ${ }^{\circledR}$ HLA SSO Typing Kit (Gen-Probe, Inc.) (Later sequenced by Allele SEQR ${ }^{\circledR}$ HLA PCR/Sequencing Kit, Abbott Molecular) were analyzed by our SSP-PCR.

HLA-A BW4 and HLA-B BW4- $\mathrm{I}^{80} / \mathrm{T}^{80}$ were identified under the same conditions as HLA-C. Validation for HLA-A and B was also performed by selected samples previously genotyped by high

\section{TABLE 1 | KIR genotyping primers.}

\begin{tabular}{|c|c|c|c|c|c|}
\hline KIR & Forward primers $\left(5^{\prime}-3^{\prime}\right)$ & Amplicon (bp) & Annealing temp $\left({ }^{\circ} \mathrm{C}\right)$ & Control gene & Alleles not detected \\
\hline 2DS2 & $\begin{array}{l}\text { CGGGCCCCACGGTT } \\
\text { GGTCACTCGAGTTGACCACTCA }\end{array}$ & 240 & 60 & CRP & \\
\hline 2DS4 & $\begin{array}{l}\text { TAGGCTCCCTGCAGTGCG } \\
\text { GAGTTTACCACTCGTAGGGAGC }\end{array}$ & 129 & 63 & CAMP & $\begin{array}{l}2 \mathrm{DS} 4^{*} 003 \\
2 \mathrm{DS} 4^{*} 004 \\
2 \mathrm{DS} 4^{*} 006 \\
2 \mathrm{DS} 4^{*} 007 \\
2 \mathrm{DS} 4^{*} 009\end{array}$ \\
\hline 2DS1 & $\begin{array}{l}\text { CTTCTCCATCAGTCGCAT } \\
\text { AGGGTCACTGGGAGCTGACAA }\end{array}$ & 102 & 53 & CAMP & \\
\hline 3DS1 & $\begin{array}{l}\text { GCCCAGCGCTGTGGTGCCTCGC } \\
\text { CTGCAAGGGCACGCATCATGGA }\end{array}$ & 1958 & 71 & CAMP & $3 \mathrm{DS} 1^{\star} 047$ \\
\hline 2DL2 & $\begin{array}{l}\text { CTGGCCCACCCAGGTCG } \\
\text { GGACCGATGGAGAAGTTGGCT }\end{array}$ & 173 & 60 & CRP & $2 \mathrm{DL} 2{ }^{*} 004$ \\
\hline 3DL2 & $\begin{array}{l}\text { CCCATGAACGTAGGCTCCG } \\
\text { CACACGCAGGGCAGGG }\end{array}$ & 130 & 54 & CRP & \\
\hline
\end{tabular}

Expected PCR products, annealing temperature $\left({ }^{\circ} \mathrm{C}\right)$, and allelic specificity are given per pair of primers. Primer reference is (22) for all KIRs except $2 \mathrm{DS} 1$, for which the primer reference is (23). 
resolution methods. All primer sequences [based on Hong et al. (23)] and expected PCR products for HLA-A, -B, and -C genotyping are listed in Table 2.

All amplified SSP-PCR products were visualized by agarose gel electrophoresis using a non-mutagenic fluorescent reagent ("Novel Juice"; GeneDireX, Las Vegas City, NV, USA). Selected sample from each amplified band was sent for sequencing.

\section{Statistical Analysis}

The Wilcoxon Signed-Ranks test $(p<0.05)$ was determined using SPSS software. When combination of more than one factor to specific receptor was detected, some different aspects were detected. The first refers to all ligands equally, and compares the sum of all ligands (relevant to specific receptor) found in each person to determine the mean value. This is defined as "Ligand Composite" (Table 7). Secondly, an alternative designation of "Ligand One+" compares existence of at least one ligand to this specific receptor. This was the case in C1 + 2DL2/2DL3/2DS2, C2 + 2DL1/2DS1 (Table 8). In contrast, for overall signaling (Table 9) and in the case of A_BW4 + 3DL1/3DS1 and B_BW4 + 3DL1/3DS1 (Table 7) only the first option is shown. All HLAB genotyping analysis is described as "total," meaning having $\mathrm{I}^{80} / \mathrm{T}^{80}$.

\section{RESULTS}

\section{HLA and KIRs Frequency Comparison}

Comparison of the nine different KIR genes and HLA frequencies revealed several main differences between parents of autistic children (Tables 3-5).

The most surprising finding is higher frequency of HLAC2 allotypes in fathers of ASD children (mean 0.73 vs. 0.52 , $p=0.038)$, in contrast to $\mathrm{C} 1$ frequencies, which were the same in both mothers and fathers ( 0.88 vs. 0.91$)$ (Table 5). On the other hand, 2DS1, which is known to interact specifically with HLA-C2, was found in higher percentage $(0.55$ vs. $0.34, p=0.022)$ in the maternal group (Table 4). Additionally 3DS1, with its specificity against BW4 allotypes, was found to be higher in mothers than in their ASD children $(0.51$ vs. $0.36, p=0.044)$ (Table 4). Other KIR frequencies (2DL2, 2DL1, 2DL3, 3DL1, 3DL2, 2DS2, and 2DS4)

\begin{tabular}{|c|c|c|c|}
\hline \multirow{2}{*}{$\begin{array}{l}\text { HLA } \\
\text { allotype }\end{array}$} & \multirow[t]{2}{*}{ Direction } & Forward primers $\left(5^{\prime}-3^{\prime}\right)$ & \multirow{2}{*}{$\begin{array}{c}\text { Amplicon } \\
\quad \text { (bp) }\end{array}$} \\
\hline & & Reverse primers $\left(5^{\prime}-3^{\prime}\right)$ & \\
\hline \multirow[t]{3}{*}{$\mathrm{HLA}-\mathrm{C}$} & Forward & CGCCGCGAGTCCGAGAGG & 139 \\
\hline & Reverse (C1: N80) & GTTGTAGTAGCCGCGCAGG & \\
\hline & Reverse (C2: $\left.\mathrm{K}^{80}\right)$ & GTTGTAGTAGCCGCGCAGT & \\
\hline HLA-A & Forward & CCATTGGGTGTCGGGTTTC & 569 \\
\hline BW4:180 & Reverse & CTCTGGTTGTAGTAGCGGAGCGCG & \\
\hline HLA-B & Forward & ACCCGGACTCAGAATCTCC & 366 \\
\hline BW4:180 & Reverse & CTCTGGTTGTAGTAGCGGAGCGCG & \\
\hline HLA-B & Forward & ACCCGGACTCAGAATCTCC & 369 \\
\hline BW4:T80 & Reverse & CTCTGGTTGTAGTAGCGGAGCAGG & \\
\hline
\end{tabular}

Expected PCR product sizes are given. In the case of HLA-C, forward primer was either paired with the $C 1\left(N^{80}\right)$ or $C 2\left(K^{80}\right)$ reverse primers, leading to an amplification product of $139 \mathrm{bp}$.

$F$, forward; $R$, reverse. were similar in both mothers and fathers, and corresponded with values expected in Asian populations. ${ }^{2}$ Most KIR and HLA frequencies in ASD children were similar to those detected in parents.

Positive correlation between parent (mother or father) and child, reflecting the likelihood of transferring the gene (Tables 3-5) were found relative to most of genes, which may reflect a high probability of homozygous genotyping for these genes.

\section{KIR Signaling Through HLA Molecules}

Relevant combinations between KIR receptors and their specific HLA ligands were detected (Table 6). ASD children did not exhibit

${ }^{2}$ http://www.allelefrequencies.net/kir6008a.asp

TABLE 3 | Inhibitory KIR frequencies in Israeli families with at least 1 ASD-diagnosed child.

\begin{tabular}{|c|c|c|c|c|c|c|c|}
\hline \multicolumn{2}{|c|}{ Inhibitory KIR } & \multirow{2}{*}{$\begin{array}{r}\text { Child }^{\text {ASD }} \\
0.59\end{array}$} & \multirow{2}{*}{$\begin{array}{r}\text { Father } \\
0.63\end{array}$} & \multirow{2}{*}{$\begin{array}{r}\text { Child }^{\text {ASD }} \\
0.58\end{array}$} & \multirow{2}{*}{$\begin{array}{r}\text { Mother } \\
0.66\end{array}$} & \multirow{2}{*}{$\begin{array}{c}\text { Father } \\
0.64\end{array}$} & \multirow{2}{*}{$\begin{array}{c}\text { Mother } \\
0.68\end{array}$} \\
\hline \multirow[t]{3}{*}{ 2DL2 } & Mean & & & & & & \\
\hline & $Z$ & \multicolumn{2}{|c|}{-0.577} & \multicolumn{2}{|c|}{-0.894} & \multicolumn{2}{|c|}{-0.408} \\
\hline & Pearson's $r$ & \multicolumn{2}{|c|}{$0.487^{\star}$} & \multicolumn{2}{|c|}{0.204} & \multicolumn{2}{|c|}{ NR } \\
\hline \multirow[t]{3}{*}{ 2DL1 } & Mean & 0.96 & 0.98 & 0.96 & 0.96 & 0.98 & 0.95 \\
\hline & Z & \multicolumn{2}{|c|}{-1} & \multicolumn{2}{|c|}{0} & \multicolumn{2}{|c|}{-1} \\
\hline & Pearson's $r$ & \multicolumn{2}{|c|}{$0.7^{\star}$} & \multicolumn{2}{|c|}{-0.039} & \multicolumn{2}{|c|}{ NR } \\
\hline \multirow[t]{3}{*}{ 2DL3 } & Mean & 0.86 & 0.84 & 0.87 & 0.89 & 0.84 & 0.88 \\
\hline & Z & \multicolumn{2}{|c|}{-0.302} & \multicolumn{2}{|c|}{-0.333} & \multicolumn{2}{|c|}{-0.535} \\
\hline & Pearson's $r$ & \multicolumn{2}{|c|}{0.135} & \multicolumn{2}{|c|}{0.212} & \multicolumn{2}{|c|}{ NR } \\
\hline \multirow[t]{3}{*}{ 3DL1 } & Mean & 0.94 & 0.94 & 0.94 & 0.89 & 0.93 & 0.89 \\
\hline & Z & \multicolumn{2}{|c|}{0} & \multicolumn{2}{|c|}{-1.342} & \multicolumn{2}{|c|}{-0.707} \\
\hline & Pearson's $r$ & \multicolumn{2}{|c|}{$0.645^{\star}$} & \multicolumn{2}{|c|}{$0.428^{\star}$} & \multicolumn{2}{|c|}{ NR } \\
\hline \multirow[t]{3}{*}{ 3DL2 } & Mean & 1 & 1 & 1 & 1 & 1 & 1 \\
\hline & Z & \multicolumn{2}{|c|}{0} & & 0 & & 0 \\
\hline & Pearson's $r$ & & $\mathrm{JR}$ & & NR & & $\mathrm{JR}$ \\
\hline
\end{tabular}

$Z=$ Wilcoxon Signed-Ranks Test value

$N R=$ not relevant

${ }^{*} p \leq 0.01$

TABLE 4 | Activating KIR frequencies in Israeli families with at least 1 ASD-diagnosed child.

\begin{tabular}{|c|c|c|c|c|c|c|c|}
\hline \multicolumn{2}{|c|}{ Activating KIR } & \multirow{2}{*}{$\begin{array}{c}\text { Child }^{\text {ASD }} \\
0.61\end{array}$} & \multirow{2}{*}{$\begin{array}{r}\text { Father } \\
0.63\end{array}$} & \multirow{2}{*}{$\begin{array}{c}\text { Child }^{\text {ASD }} \\
0.6\end{array}$} & \multirow{2}{*}{$\begin{array}{r}\text { Mother } \\
0.62\end{array}$} & \multirow{2}{*}{\multicolumn{2}{|c|}{$\begin{array}{rr}\text { Father } & \text { Mother } \\
0.64 & 0.66\end{array}$}} \\
\hline \multirow[t]{3}{*}{ 2DS2 } & Mean & & & & & & \\
\hline & $Z$ & \multicolumn{2}{|c|}{-0.302} & \multicolumn{2}{|c|}{-0.209} & \multicolumn{2}{|c|}{-0.209} \\
\hline & Pearson's $r$ & \multicolumn{2}{|c|}{$0.523^{\star \star}$} & \multicolumn{2}{|c|}{0.086} & \multicolumn{2}{|c|}{ NR } \\
\hline \multirow[t]{3}{*}{ 2DS4 } & Mean & 0.96 & 0.96 & 0.94 & 0.89 & 0.94 & 0.91 \\
\hline & $Z$ & \multicolumn{2}{|c|}{0} & \multicolumn{2}{|c|}{-1.342} & \multicolumn{2}{|c|}{-0.707} \\
\hline & Pearson's $r$ & \multicolumn{2}{|c|}{$0.478^{\star \star}$} & \multicolumn{2}{|c|}{$0.428^{* \star}$} & \multicolumn{2}{|c|}{ NR } \\
\hline \multirow[t]{3}{*}{ 2DS1 } & Mean & 0.51 & 0.37 & 0.49 & 0.57 & 0.34 & 0.55 \\
\hline & Z & \multicolumn{2}{|c|}{-1.528} & \multicolumn{2}{|c|}{-1.069} & \multicolumn{2}{|c|}{$-2.268^{\star}$} \\
\hline & Pearson's $r$ & \multicolumn{2}{|c|}{0.154} & \multicolumn{2}{|c|}{$0.478^{\star \star}$} & \multicolumn{2}{|c|}{ NR } \\
\hline \multirow[t]{3}{*}{ 3DS1 } & Mean & 0.37 & 0.39 & 0.36 & 0.51 & 0.39 & 0.5 \\
\hline & Z & \multicolumn{2}{|c|}{-0.728} & \multicolumn{2}{|c|}{$-2^{*}$} & \multicolumn{2}{|c|}{-1.279} \\
\hline & Pearson's $r$ & \multicolumn{2}{|c|}{$0.281^{\star}$} & \multicolumn{2}{|c|}{$0.419^{\star \star}$} & \multicolumn{2}{|c|}{ NR } \\
\hline
\end{tabular}

$Z=$ Wilcoxon Signed-Ranks Test value.

$N R=$ not relevant.

${ }^{*} p \leq 0.05$.

${ }^{* *} p \leq 0.01$ 
TABLE 5 | HLA ligand frequencies in Israeli families with at least 1 ASD-diagnosed child.

\begin{tabular}{|c|c|c|c|c|c|c|c|c|}
\hline HLA Ligand & & ChildASD $^{\text {AsD }}$ & Father & ChildASD $^{\text {ASD }}$ & Mother & Father & & Mother \\
\hline \multirow[t]{3}{*}{ HLAC1 } & Mean & 0.86 & 0.9 & 0.83 & 0.85 & 0.88 & & 0.91 \\
\hline & Z & & & \multicolumn{2}{|c|}{-0.333} & \multicolumn{3}{|c|}{-0.577} \\
\hline & Pearson's $r$ & & & \multicolumn{2}{|c|}{$0.371^{* \star}$} & \multicolumn{3}{|c|}{ NR } \\
\hline \multirow[t]{3}{*}{ HLAC2 } & Mean & 0.65 & 0.72 & 0.68 & 0.57 & 0.73 & & 0.52 \\
\hline & Z & & & \multicolumn{2}{|c|}{-1.342} & \multicolumn{3}{|c|}{$-2.058^{*}$} \\
\hline & Pearson's $r$ & & & \multicolumn{2}{|c|}{0.214} & \multicolumn{3}{|c|}{ NR } \\
\hline \multirow{3}{*}{ HLA-A-BW4 } & Mean & 0.33 & 0.37 & 0.3 & 0.28 & 0.36 & & 0.3 \\
\hline & Z & & & \multicolumn{2}{|c|}{-0.229} & \multicolumn{3}{|c|}{-0.557} \\
\hline & Pearson's $r$ & & & \multicolumn{2}{|c|}{0.134} & \multicolumn{3}{|c|}{ NR } \\
\hline \multirow[t]{3}{*}{ HLA-B-BW4 } & Mean & 0.88 & 0.86 & 0.88 & 0.81 & 0.87 & & 0.8 \\
\hline & $Z$ & & & \multicolumn{2}{|c|}{1.272} & \multicolumn{3}{|c|}{1} \\
\hline & Pearson's $r$ & & & \multicolumn{2}{|c|}{$0.248^{\star}$} & \multicolumn{3}{|c|}{ NR } \\
\hline
\end{tabular}

$Z=$ Wilcoxon Signed-Ranks Test value

$N R=$ not relevant

${ }^{*} p \leq 0.05$.

${ }^{* *} \mathrm{p} \leq 0.01$.

TABLE 6 | Frequency of each KIR detected and its corresponding ligand in Israeli ASD families, consisting of both parents and an ASD-diagnosed child.

\begin{tabular}{|c|c|c|c|c|c|}
\hline KIR & & ChildASD & Father & ChildASD & Father \\
\hline \multirow[t]{2}{*}{ 2DL2 + C1 } & Z & -0.832 & & -1.342 & -1.177 \\
\hline & Pearson's $r$ & $0.474^{\star \star}$ & & 0.259 & NR \\
\hline \multirow[t]{2}{*}{ 2DL1 + C2 } & Z & -0.535 & & -1.091 & -1.859 \\
\hline & Pearson's $r$ & $0.372^{\star \star}$ & & 0.176 & NR \\
\hline \multirow{2}{*}{ 2DL3 + C1 } & Z & 0 & & 0 & -0.426 \\
\hline & Pearson's $r$ & 0.227 & & $0.321^{*}$ & NR \\
\hline \multirow[t]{2}{*}{ 3DL1 + A_BW4 } & Z & -0.227 & & -0.243 & -0.392 \\
\hline & Pearson's $r$ & 0.148 & & 0.156 & NR \\
\hline \multirow{2}{*}{ 3DL1 + B_BW4 } & $Z$ & -0.302 & & $-1.941^{\star}$ & -1.225 \\
\hline & Pearson's $r$ & $0.283^{\star}$ & & $0.359^{\star \star}$ & NR \\
\hline \multirow{2}{*}{$2 \mathrm{DS} 2+\mathrm{C} 1$} & Z & -0.577 & & -0.853 & -1 \\
\hline & Pearson's $r$ & $0.511^{\star \star}$ & & 0.174 & NR \\
\hline \multirow{2}{*}{ 2DS4 + A_BW4 } & $Z$ & -0.577 & & -0.243 & -0.6 \\
\hline & Pearson's $r$ & $0.393^{\star *}$ & & 0.156 & NR \\
\hline \multirow{2}{*}{ 2DS4 + B_BW4 } & $Z$ & -0.302 & & $-1.941^{\star}$ & -1.279 \\
\hline & Pearson's $r$ & 0.211 & & $0.359^{\star \star}$ & NR \\
\hline \multirow{2}{*}{$2 \mathrm{DS} 1+\mathrm{C} 2$} & $Z$ & -0.243 & & -0.775 & -0.784 \\
\hline & Pearson's $r$ & 0.132 & & $0.345^{\star \star}$ & NR \\
\hline \multirow{2}{*}{ 3DS1 + A_BW4 } & $Z$ & 0 & & -0.577 & -0.535 \\
\hline & Pearson's $r$ & $0.456^{\star \star}$ & & 0.12 & NR \\
\hline \multirow[t]{2}{*}{ 3DS1 + B_BW4 } & Z & -0.728 & & -1.414 & -0.853 \\
\hline & Pearson's $r$ & 0.25 & & $0.295^{\star}$ & NR \\
\hline
\end{tabular}

Z = Wilcoxon Signed-Ranks Test value.

$N R$, not relevant.

${ }^{*} p \leq 0.05$.

${ }^{* *} p \leq 0.01$.

high frequency of the combination HLA-C2 + KIR2DS1, despite elevated paternal HLA-C2 and maternal 2DS1 frequencies.

Correlational analysis between parent (mother or father) and child reflected the likelihood of transferring a gene with its corresponding ligand. Significant values were reported in most cases (Table 6). It is important to mention, these gene families are located on different autosomal chromosomes, supporting homozygous status for most of the genes.

The next evaluation involves the examination of particular HLA ligands with presence/absence of all relevant activating/ inhibitory KIR genes (Table 7). "Ligand Composite" analysis (see
Materials and Methods) was compared between ASD children and their parents, as well as "Ligand One+" values (Table 8). Significant differences were found between parental groups in the case of C2 + 2DL1/2DS1 "Ligand One+" $(p=0.040)$.

\section{Overall Balance of Activation and Inhibition}

Final analysis (Table 9) presents a full view of the genotyping. It may allow insight to the overall expected interaction of KIR/ HLA in Israeli autistic individuals and their non-autistic parents, 
TABLE 7 | Comparison of particular HLA ligands with presence/absence of all relevant activating/inhibitory KIR genes in Israeli ASD families.

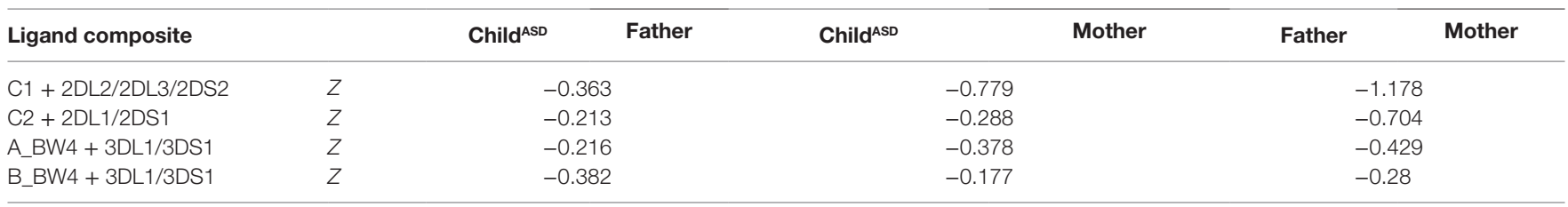

Z = Wilcoxon Signed-Ranks Test value.

TABLE 8 | Interactions of KIR and HLA in autistic Israeli patients and their parents, based on overall genotype mapping.

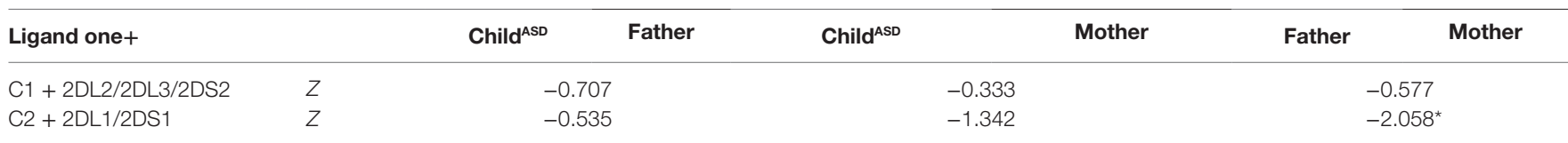

$Z=$ Wilcoxon Signed-Ranks Test value.

${ }^{*} p \leq 0.05$.

TABLE 9 | Overall ligand and interaction summaries.

\begin{tabular}{|c|c|c|c|c|c|c|c|}
\hline & & Child $^{\text {ASD }}$ & Father & Child ASD & Mother & Father & Mother \\
\hline \multirow[t]{3}{*}{ KIRs: activating } & Mean & 2.4043 & 2.3191 & 2.3962 & 2.5849 & 2.2593 & 2.6667 \\
\hline & Pearson's $r$ & \multicolumn{2}{|c|}{$0.331^{*}$} & \multicolumn{2}{|c|}{$0.274^{\star}$} & \multicolumn{2}{|c|}{0.104} \\
\hline & $t$-Test & \multicolumn{2}{|c|}{0.443} & \multicolumn{2}{|c|}{-1.121} & \multicolumn{2}{|c|}{$-2.015^{\star}$} \\
\hline \multirow[t]{3}{*}{ KIRs: inhibitory } & Mean & 4.3469 & 4.3878 & 4.3585 & 4.3962 & 4.4 & 4.3818 \\
\hline & Pearson's $r$ & \multicolumn{2}{|c|}{0.229} & \multicolumn{2}{|c|}{-0.023} & \multicolumn{2}{|c|}{0.153} \\
\hline & $t$-Test & \multicolumn{2}{|c|}{-0.362} & \multicolumn{2}{|c|}{-0.286} & \multicolumn{2}{|c|}{0.155} \\
\hline \multirow[t]{3}{*}{ Interactions: activating } & Mean & 1.9149 & 1.9787 & 1.9245 & 2.0377 & 1.9259 & 2.1111 \\
\hline & Pearson's $r$ & \multicolumn{2}{|c|}{0.216} & \multicolumn{2}{|c|}{0.142} & \multicolumn{2}{|c|}{-0.119} \\
\hline & $t$-Test & \multicolumn{2}{|c|}{-0.308} & \multicolumn{2}{|c|}{-0.636} & \multicolumn{2}{|c|}{-0.778} \\
\hline \multirow[t]{3}{*}{ Interactions: inhibitory } & Mean & 2.6939 & 2.7959 & 2.6981 & 2.6038 & 2.8214 & 2.6607 \\
\hline & Pearson's $r$ & \multicolumn{2}{|c|}{0.272} & \multicolumn{2}{|c|}{0.114} & \multicolumn{2}{|c|}{-0.099} \\
\hline & $t$-Test & \multicolumn{2}{|c|}{-0.647} & \multicolumn{2}{|c|}{0.582} & \multicolumn{2}{|c|}{0.903} \\
\hline
\end{tabular}

${ }^{*} p \leq 0.05$.

${ }^{* *} p \leq 0.01$.

based on all genotype mapping. Mothers have significantly higher levels of activating signals than their male counterparts $(p=0.049)$.

\section{DISCUSSION}

Despite the increased attention garnered by autism research in recent years, the condition is currently diagnosed only after the infant reaches 2 or 3 years of age. Findings in autistic patients indicate multifactorial inheritance, while definitive genetic factors can be diagnosed in only a small percentage of cases. Immunological studies revealed a unique and distinguishing profile in ASD patients compared to controls. In the context of NK cells, altered gene expression, absolute number and function have been reported $(7,24,25)$. Reports of modified NK function led us to investigate the highly polymorphic cell surface proteins on NK cells, called KIRs, to search for genetic background underlying the immune abnormalities. By interacting with MHC class I proteins expressed on all cell types, KIRs regulate the killing function of NK.
The most significant finding in this study is the unequal distribution of HLA-C2 (higher in fathers of ASD children) known to interact with KIR 2DS1 (higher in mothers of ASD children) to activate NK function. Analysis of "overall activation" (Table 9) signaling pointed to higher activation in maternal than paternal cohorts. Most HLA and KIR frequencies in ASD children ranged between those of their parents, indicating regular independent genetic inheritance from parents to children. This reinforced the importance of the specific HLA-KIR combinations between parents, though the mechanisms involved are still unclear. Our limitation in interpreting these findings lies in the absence of unusual prevalence of these genes or their combination in the ASD children, in comparison to their healthy parents.

Some reports correlated these initial interactions between parental cells as a possible mechanism for subsequent disease development or other complications. Hiby et al. (26) reported an increased HLA-C2 frequency in different cohorts of affected pregnancies: (a) recurrent miscarriage (RM) couples and their conception products, (b) fetal growth restriction (FGR), and (c) preeclampsia. Conversely, lack of activating KIR (in women affected by RM) or increased inhibitory KIR (in women with 
preeclampsia) $(26,27)$ has already been reported. Specifically, KIR 2DS1 seemed to be protective because of its significant lack in RM women. We find this plausible, since activating KIR 2DS1 compete with inhibitory KIR 2DL1 in interaction with HLA-C2, such that a predominance of KIR 2DS1 can overcome the strong inhibitory effect mediated by KIR 2DL1. All these findings support the importance of the innate immune system (mediated by maternal uterine NK and fetal trophoblast cells), modulated by KIR-HLA interaction to play a beneficial role in reproductive success.

Some papers have already pointed out differential segregation of KIR-HLA genes in autistic patients. Our study expands upon this by focusing within ASD families. Torres et al. (28) examined a large autistic cohort, revealing a highly significant increase in the activating KIR gene 2DS1 and its cognate HLA-C2 ligand. This increase in activating KIR may explain, at least partially, the high frequency of autoimmune diseases in autism patients. Guerini et al. (29) compared ASD children to their non-ASD mothers and showed both that activating KIR/HLA combinations were increased and inhibitory KIR/HLA were reduced in ASD children. This difference was proposed as having resulted from the genetic influence of the mothers, as these molecules' expression and interaction are relevant in the surrounding uterus.

Ethnic populations are known to differ in KIR genotype frequencies and genotype content (30). To the best of our knowledge, this is the first study to research KIR and HLA combinations in the Israeli Jewish population. As shown in the Allele Frequency Net Database, ${ }^{3}$ our geographical location has higher frequency of HLA-C2 (around 0.4-0.7 in Africa and the Middle East). However, we showed here a significant skewing between maternal and paternal $\mathrm{C} 2$ frequencies. It raises an interesting question, since HLA clusters map in autosomal chromosomes, leading to independent segregation without respect to gender.

Genotype mapping of this system has certain limitations. While theoretically all gene segments identified by our SSPPCR system may be expressed on the cell surface (meaning the functional KIR repertoire may depend on the KIR genotype), the transcription and translation processes may lead to different phenotype profiles. Another limitation of the SSP-PCR method in this context is the inability to determine the heterozygous/ homozygous status of each of these KIRs. Our future plans are to determine not only genotype, but also RNA and protein levels. Another point to take into account is that NK cell function is a very complicated process determined upon the balance of an array of receptors, of which KIR is only one family. Moreover, the affinity of these interactions may differ (e.g., KIR 2DL3: HLA-C1 interaction is thought to be weak, compared to KIR 2DL2:HLA-C1 or KIR 2DL1:HLA-C2), such that one interaction may wield greater influence than another. It is also becoming increasingly clear that genetic factors, such as promoters and epigenetic mechanisms, are important in controlling NK cell receptor expression and function.

This study comprised a relatively small cohort of ASDaffected families - though statistically verified based on the

${ }^{3} \mathrm{http}: / /$ www.allelefrequencies.net/ minor allele frequency (MAF) of C2 (39.5\%). In the absence of a control group, our findings were compared with the general population data of our geographical area. Additionally, the clinical patient data have not been thoroughly listed here, places limitations upon the reader in drawing parallels to other patient cohorts. The trends revealed in this preliminary investigation need to be further corroborated in ongoing and expanded research efforts.

Finally, we would like to emphasis another interesting aspect. On the one hand, different pregnancy complications (FGR, spontaneous abortion, placental abruption) are known to stem (at least to some degree) from immunological factors. On the other hand, perinatal factors unrelated to the immunological milieu may also be important in the pathogenesis of ASD. Certain complications during gestation were found to occur with increased frequency in mothers of ASD patients; an immunological basis has been suggested as underlying a portion of these. In some cases, intermediate factors may play a partial role in the association (e.g., reduced birth weight).

\section{CONCLUSION}

The aim of our research was to genotype the KIR receptors and their relevant HLA ligands in autistic children and their non-autistic parents, in order to screen for possible unique combinations. The most interesting finding in this study is higher frequency of HLAC2 allotypes in the paternal group besides higher percentage of KIR 2DS1 in the maternal group. Such interaction may lead to NK activation. An absence of unusual prevalence of these genes or their combination in the ASD children, limited our ability to interpret these findings. Moreover, analysis of "overall activation" signaling pointed to higher activation in maternal than paternal cohorts.

\section{AUTHOR CONTRIBUTIONS}

Mrs. MG is a Ph.D. student. She is the major contributor to this manuscript doing the thinking, bench work, and manuscript writing. Mrs. KA contributed to the bench work as well as writing the manuscript. Prof. NY is an initiator of the original study and has performed the clinical diagnosis of all the participants of the study. Prof. RE is the initiator of original study recruited the families and the original head of the lab. Dr. DM is the head of the lab the study was conducted in, supported the study, and contributed to the manuscript writing and editing.

\section{ACKNOWLEDGMENTS}

This work was supported by a research grant (\#232-12-13B) from the National Institute for Psychobiology in Israel, founded by the Charles E. Smith Family, and the Harris Foundation. The authors wish to thank Mrs. Shoshana Israel, Ph.D., and Mrs. Amal Halabi, M.Sc., for their help with validating HLA primers, and Dr. Lubov Nemanov for her assistance in processing patient samples. MG was supported by the Hoffman Leadership and Responsibility Fund, at the Hebrew University. 


\section{REFERENCES}

1. Caronna EB, Milunsky JM, Tager-Flusberg H. Autism spectrum disorders: clinical and research frontiers. Arch Dis Child (2008) 93:518-23. doi:10.1136/ adc. 2006.115337

2. Blumberg SJ, Bramlett MD, Kogan MD, Schieve LA, Jones JR, Lu MC. Changes in prevalence of parent-reported autism spectrum disorder in school-aged U.S. children: 2007 to 2011-2012. Natl Health Stat Report (2013) 1-11:1 following11.

3. Persico AM, Napolioni V. Autism genetics. Behav Brain Res (2013) 251:95-112. doi:10.1016/j.bbr.2013.06.012

4. Geschwind DH. Genetics of autism spectrum disorders. Trends Cogn Sci (2011) 15:409-16. doi:10.1016/j.tics.2011.07.003

5. Hu VW. From genes to environment: using integrative genomics to build a "systems-level" understanding of autism spectrum disorders. Child Dev (2013) 84:89-103. doi:10.1111/j.1467-8624.2012.01759.x

6. Noriega DB, Savelkoul HF. Immune dysregulation in autism spectrum disorder. Eur J Pediatr (2014) 173:33-43. doi:10.1007/s00431-013-2183-4

7. Enstrom AM, Lit L, Onore CE, Gregg JP, Hansen RL, Pessah IN, et al. Altered gene expression and function of peripheral blood natural killer cells in children with autism. Brain Behav Immun (2009) 23:124-33. doi:10.1016/j. bbi.2008.08.001

8. Biassoni R. Natural killer cell receptors. Adv Exp Med Biol (2008) 640:35-52. doi:10.1007/978-0-387-09789-3_4

9. Rajalingam R, Ashouri E. Gene-specific PCR typing of killer cell immunoglobulin-like receptors. Methods Mol Biol (2013) 1034:239-55. doi:10.1007/978-1-62703-493-7_12

10. Jamil KM, Khakoo SI. KIR/HLA interactions and pathogen immunity. J Biomed Biotechnol (2011) 2011:298348. doi:10.1155/2011/209348

11. De Re V, Caggiari L, De Zorzi M, Toffoli G. KIR molecules: recent patents of interest for the diagnosis and treatment of several autoimmune diseases, chronic inflammation, and B-cell malignancies. Recent Pat DNA Gene Seq (2011) 5:169-74. doi:10.2174/187221511797636266

12. Kulkarni S, Martin MP, Carrington M. The Yin and Yang of HLA and KIR in human disease. Semin Immunol (2008) 20:343-52. doi:10.1016/j. smim.2008.06.003

13. Holm SJ, Sakuraba K, Mallbris L, Wolk K, Stahle M, Sanchez FO. Distinct HLA-C/KIR genotype profile associates with guttate psoriasis. I Invest Dermatol (2005) 125:721-30. doi:10.1111/j.0022-202X.2005.23879.x

14. Zhi D, Sun C, Sedimbi SK, Luo F, Shen S, Sanjeevi CB. Killer cell immunoglobulin-like receptor along with HLA-C ligand genes are associated with type 1 diabetes in Chinese Han population. Diabetes Metab Res Rev (2011) 27:872-7. doi:10.1002/dmrr.1264

15. Barker DJ, Osmond C, Golding J, Kuh D, Wadsworth ME. Growth in utero, blood pressure in childhood and adult life, and mortality from cardiovascular disease. BMJ (1989) 298:564-7. doi:10.1136/bmj.298.6673.564

16. Gamliel M, Ebstein R, Yirmiya N, Mankuta D. Minor fetal sonographic findings in autism spectrum disorder. Obstet Gynecol Surv (2012) 67:176-86. doi:10.1097/OGX.0b013e31824bb5d6

17. Hagberg H, Gressens P, Mallard C. Inflammation during fetal and neonatal life: implications for neurologic and neuropsychiatric disease in children and adults. Ann Neurol (2012) 71:444-57. doi:10.1002/ana.22620

18. Bashirova AA, Martin MP, McVicar DW, Carrington M. The killer immunoglobulin-like receptor gene cluster: tuning the genome for defense. Annu Rev Genomics Hum Genet (2006) 7:277-300. doi:10.1146/annurev. genom.7.080505.115726
19. Chazara O, Xiong S, Moffett A. Maternal KIR and fetal HLA-C: a fine balance. J Leukoc Biol (2011) 90:703-16. doi:10.1189/jlb.0511227

20. Xiong S, Sharkey AM, Kennedy PR, Gardner L, Farrell LE, Chazara O, et al. Maternal uterine NK cell-activating receptor KIR2DS1 enhances placentation. J Clin Invest (2013) 123:4264-72. doi:10.1172/JCI68991

21. Patterson PH. Immune involvement in schizophrenia and autism: etiology, pathology and animal models. Behav Brain Res (2009) 204:313-21. doi:10.1016/j.bbr.2008.12.016

22. Chainonthee W, Bottcher G, Gagne K, Fussel M, Bignon JD, Wassmuth R. Improved KIR gene and HLA-C KIR ligand sequence-specific primer polymerase chain reaction genotyping using whole genome amplification. Tissue Antigens (2010) 76:135-43. doi:10.1111/j.1399-0039.2010.01479.x

23. Hong HA, Loubser AS, de Assis Rosa D, Naranbhai V, Carr W, Paximadis $\mathrm{M}$, et al. Killer-cell immunoglobulin-like receptor genotyping and HLA killer-cell immunoglobulin-like receptor-ligand identification by real-time polymerase chain reaction. Tissue Antigens (2011) 78:185-94. doi:10.1111/j.1399-0039.2011.01749.x

24. Ashwood P, Corbett BA, Kantor A, Schulman H, Van de Water J, Amaral DG. In search of cellular immunophenotypes in the blood of children with autism. PLoS One (2011) 6:e19299. doi:10.1371/journal.pone.0019299

25. Vojdani A, Mumper E, Granpeesheh D, Mielke L, Traver D, Bock K, et al. Low natural killer cell cytotoxic activity in autism: the role of glutathione, IL-2 and IL-15. J Neuroimmunol (2008) 205:148-54. doi:10.1016/j. jneuroim.2008.09.005

26. Hiby SE, Walker JJ, O'Shaughnessy KM, Redman CW, Carrington M, Trowsdale J, et al. Combinations of maternal KIR and fetal HLA-C genes influence the risk of preeclampsia and reproductive success. J Exp Med (2004) 200:957-65. doi:10.1084/jem.20041214

27. Flores AC, Marcos CY, Paladino N, Arruvito L, Williams F, Middleton D, et al. KIR receptors and HLA-C in the maintenance of pregnancy. Tissue Antigens (2007) 69(Suppl 1):112-3. doi:10.1111/j.1399-0039.2006.762_8.x

28. Torres AR, Westover JB, Givvons C, Johnson RC, Ward DC. Activating killer-cell immunoglobulin-like receptors (KIR) and their cognate HLA ligands are significantly increased in autism. Brain Behav Immun (2012) 26:1122-7. doi:10.1016/j.bbi.2012.07.014

29. Guerini FR, Bolognesi E, Chiappedi M, Manca S, Ghezzo A, Agliardi C, et al Activating KIR molecules and their cognate ligands prevail in children with a diagnosis of ASD and in their mothers. Brain Behav Immun (2014) 36:54-60. doi:10.1016/j.bbi.2013.10.006

30. Takeshita LY, Gonzalez-Galarza FF, dos Santos EJ, Maia MH, Rahman MM Zain SM, et al. A database for curating the associations between killer cell immunoglobulin-like receptors and diseases in worldwide populations. Database (Oxford) (2013) 2013:bat021. doi:10.1093/database/bat021

Conflict of Interest Statement: The authors declare that the research was conducted in the absence of any commercial or financial relationships that could be construed as a potential conflict of interest.

Copyright (c) 2016 Gamliel, Anderson, Ebstein, Yirmiya and Mankuta. This is an open-access article distributed under the terms of the Creative Commons Attribution License (CC BY). The use, distribution or reproduction in other forums is permitted, provided the original author(s) or licensor are credited and that the original publication in this journal is cited, in accordance with accepted academic practice. No use, distribution or reproduction is permitted which does not comply with these terms. 